\title{
Consecuencias y retos de la sostenibilidad en relación con la discapacidad tras el COVID-19
}

\section{Consequences and challenges of sustainability in relation to disability after COVID-19}

\section{Palabras clave}

Discapacidad, sostenibilidad, COVID-19, grupos de interés, Agenda 2030.

\section{Keywords}

Disability, sustainability, COVID-19, stakeholders, 2030 Agenda.

\author{
José Antonio Martín \\ Rodríguez \\ <jamartin@bequal.es> \\ Fundación Bequal. España
}

1. Responsabilidad social corporativa y grupos de interés

Inmersos en plena pandemia del COVID-19, sin saber las consecuencias finales de la misma, parece interesante llevar a cabo una reflexión, desde el punto de vista de la incidencia en el empleo de las personas con discapacidad y en la inclusión social en general, del impacto social que esta crisis sanitaria y económica está produciendo. Además, cabría analizar cómo con la aplicación de políticas de responsabilidad social y/o sostenibilidad se puede paliar dicho impacto e identificar qué políticas se pueden poner en marcha para afrontar mejor la recuperación y la reconstrucción, específicamente en lo que concierne a las personas con discapacidad.

Como cuestión previa es necesario repasar dos conceptos: la responsabilidad social corporativa (RSC en adelante) y los grupos de interés.

Sobre la RSC evidentemente se ha escrito mucho y desde sus inicios se ha observado una constante evolución conceptual. La primera referencia o definición de RSC la encontramos en el año 2001, cuando la Unión Europea elaboró el libro verde de la RSC, titulado Fomentar un marco europeo para la responsabilidad social de las empresas. Define este concepto como "la integración voluntaria, por parte de las empresas, de las preocupaciones sociales y ambientales en sus operaciones comerciales y en las relaciones

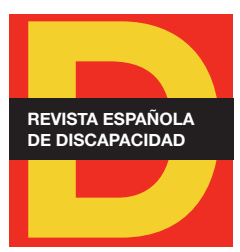

Para citar:

Martín, J. A. (2020). "Consecuencias y retos de la sostenibilidad en relación con la discapacidad tras el COVID-19". Revista Española de Discapacidad, 8(2), pp. 223-230.

Doi: <https://doi.org/10.5569/23405104.08.02.11> 
con sus interlocutores", y en un sentido más amplio como "un concepto conforme al cual las empresas deciden voluntariamente contribuir al logro de una sociedad mejor y un medio ambiente más limpio".

También cabe destacar la definición que se presenta en el acuerdo de la Comisión de Trabajo y Asuntos Sociales del Congreso de los Diputados, aprobado el 27 de junio de 2006, relativo al Informe de la Subcomisión para potenciar y promover la responsabilidad social de las empresas, el denominado Libro blanco de la $R S E$, en el que la define como "el cumplimiento estricto de las obligaciones legales vigentes, la integración voluntaria por parte de la empresa, en su gobierno y gestión, en su estrategia, políticas y procedimientos, de las preocupaciones sociales, laborales, ambientales y de respeto a los derechos humanos que surgen de la relación y el diálogo transparentes con sus grupos de interés, responsabilizándose así de las consecuencias y de los impactos que derivan de sus acciones".

Por su relevancia, no podemos obviar que la Comunicación de la Comisión Europea al Parlamento Europeo, al Consejo, al Comité Económico y Social Europeo y al Comité de las Regiones, en la Estrategia renovada de la UE para 2011-2014 sobre la Responsabilidad Social de las Empresas, de 25 de octubre de 2011, introduce un nuevo concepto donde define la RSE como "la responsabilidad de las empresas por sus impactos en la sociedad".

Actualmente el término comúnmente usado es el de "sostenibilidad"1, aunque también se utilizan términos como "propósito", "empresa con alma" o "capitalismo inclusivo".

En resumen, podemos concluir que una organización es socialmente responsable, es sostenible, cuando además del cumplimiento de sus obligaciones legales, responde de forma voluntaria y satisfactoriamente, a las expectativas que sobre su funcionamiento tienen los distintos grupos de interés en los aspectos medioambientales, sociales y de buen gobierno.

El concepto grupo de interés o stakeholder es definido por la norma-guía ISO 26000 (AENOR, 2010) como "todo individuo o grupo que tiene interés en cualquier decisión o actividad de la organización". Dicho de otro modo, el conjunto de partes interesadas y/o afectadas por la actividad de una organización. Pueden ser grupos o personas que representan a cosas o a intereses medioambientales o sociales y que afectan o son afectados, directa o indirectamente, por el desempeño de la actividad de una organización.

Se puede decir que los grupos de interés condicionan la sostenibilidad y los resultados de una organización; por ello, deben ser identificados y se debe contar con ellos no solo para descubrir nuevas oportunidades y retos sino también para anticiparse a los riesgos y estar preparados para minimizarlos.

1. El 26 de junio de 2020 la Comisión Nacional del Mercado de Valores aprobaba la revisión parcial del Código de Buen Gobierno de Sociedades Cotizadas de 2015, en el que sustituye el término RSC por "el más amplio y utilizado actualmente de sostenibilidad en relación con aspectos medioambientales, sociales y de gobierno corporativo (ESG/ASG)". 


\section{Grupo de interés y sostenibilidad son las dos caras de una misma moneda}

\subsection{La Agenda 2030 y la discapacidad}

La sostenibilidad tiene que tener muy en cuenta a los grupos vulnerables, específicamente a las personas con discapacidad, como grupo de interés relevante, tanto como empleados, como clientes, como proveedores, como inversores, como organizaciones sociales, etc. y así viene marcado en la Agenda 2030 para el desarrollo sostenible.

La Agenda 2030 es un plan de acción en favor de las personas, el planeta, la prosperidad y la paz universal, que fue aprobada el 25 de septiembre de 2015 por la Asamblea General de Naciones Unidas. Cuenta con 17 Objetivos de Desarrollo Sostenible (ODS) y 169 metas concretas a desarrollar hasta el año 2030.

Uno de los principales objetivos de la Agenda 2030 es empoderar y mejorar el bienestar de las personas que se encuentran en situación de vulnerabilidad, incluyendo dentro de este grupo a las personas con discapacidad.

En este sentido, en el preámbulo de la Agenda 2030 cuando se declara que "cuando nos embarcamos en este viaje colectivo, prometemos que nadie se quedará atrás", se hace la primera referencia a la inclusión social. Del mismo modo, en la introducción se reconoce que la dignidad de todas las personas es algo fundamental, y que los objetivos y metas se deben aplicar a todos los segmentos de la sociedad siendo prioritario empezar por las personas con mayor riesgo de exclusión, como son las personas con discapacidad.

Dentro de los indicadores, hay metas que mencionan explícitamente la discapacidad y permiten que las áreas clave en el progreso se midan sistemáticamente en relación a esta. Asimismo, en caso de brechas en el progreso que afecten a las personas con discapacidad, se facilita la identificación y la toma de medidas para reducir las desigualdades.

En total, hay 11 menciones directas a las personas con discapacidad en la Agenda 2030 y 19 en los ODS:

- 1 vez en la introducción.

- 7 veces en los objetivos.

- 11 veces en los indicadores.

Fundamentalmente estas referencias a la discapacidad se incluyen en las áreas temáticas de educación de calidad, trabajo decente y crecimiento económico, la reducción de las desigualdades, y las ciudades y comunidades sostenibles.

Además, la discapacidad se menciona de manera indirecta en numerosas ocasiones en los objetivos, metas e indicadores a través de expresiones como "para todos", "los vulnerables", "no-discriminatorios", "acceso igualitario" o "acceso universal".

En nuestro país, el Plan de Acción para la implementación de la Agenda 2030 diseñado por el Gobierno de España, aborda todos los ODS y la contribución que se hará a los mismos. Según dicho plan "la Agenda 
2030 está en el centro de la visión del Estado" y "de la acción de gobierno", y en él se mide y menciona de manera frecuente los progresos relacionados con las personas con discapacidad.

Para la sostenibilidad es fundamental la consecución de los ODS. Pero cabe preguntarse ¿es conocida la Agenda 2030? Según el barómetro del Centro de Investigaciones Sociológicas (en adelante, CIS), publicado el jueves 17 de septiembre de 2020, el 70,4 \% de los españoles no han oído hablar de la Agenda 2030.

Si todos estamos compelidos a contribuir a su cumplimiento, como forma de asegurar el progreso sostenible del planeta y de los que en el habitamos, no estamos ante un dato alentador, porque lo que no se conoce es como si no existiera. Por ello, para poder profundizar en la consecución de los objetivos hacia 2030 es necesario, en primer lugar, que se avance en su conocimiento.

\subsection{Efectos del COVID-19 en la Agenda 2030}

La crisis provocada por el COVID-19 sin duda nos aleja del cumplimiento de los ODS, ya que con la pandemia las prioridades de los gobiernos han cambiado y también las prioridades de las empresas que, en gran medida, han pasado a tener otras prioridades.

Como señalan la Red Española del Pacto Mundial, las Naciones Unidas y los diferentes expertos en desarrollo sostenible, la pandemia de COVID-19 va a tener un fuerte impacto en la Agenda 2030. Entre otros, en los ODS que se refieren al incremento de la pobreza (ODS 1), el detrimento de la salud comunitaria (ODS 3), la educación de calidad (ODS 4), el empleo decente y crecimiento económico (ODS 8) o el incremento de las desigualdades debido a la crisis económica (ODS 10).

El informe Goalkeepers (2020) que publica anualmente la Fundación Gates, y que analiza el grado de cumplimiento de los ODS señala, en referencia al ODS 1 "Fin de la pobreza", que la pandemia no solo ha estancado el progreso, sino que nos ha hecho retroceder.

Además, destaca que, desde hace dos décadas y hasta ahora, el índice de pobreza extrema² se iba reduciendo anualmente poco más de un punto. Sin embargo, este año el dato pone de relieve que ha aumentado un $7,1 \%$, lo que significa que 37 millones más de personas están viviendo con alrededor de un euro y medio al día.

En esta línea, el $9^{\circ}$ informe publicado por la Red Europea de Lucha contra la Pobreza y la Exclusión Social sobre el estado de la pobreza Seguimiento del Indicador de pobreza y exclusión social en España, 20082018 (Llano, 2019), destaca que las personas con discapacidad registran valores mucho más elevados en todas las variables de pobreza y/o exclusión y esta no es una situación coyuntural, sino que se cumple para todos los años. En el año 2018, el 31,1\%, es decir, prácticamente una de cada tres personas con discapacidad, estaba en riesgo de pobreza y/o exclusión (AROPE ${ }^{3}$ ) (Llano, 2018), 7 puntos porcentuales más que la que soportan las personas sin discapacidad.

2. Las personas en esta situación disponen de un ingreso tan bajo que, aun si lo dedicasen por completo a la adquisición de alimentos, no podrían adquirir los nutrientes necesarios para tener una vida sana.

3. AROPE: At Risk of Poverty and Exclusion (en riesgo de pobreza y exclusión). 
Por otra parte, la COVID-19 está teniendo efectos en las oportunidades de empleo de buena parte de la humanidad (OIT, 2020b).

Es evidente que la pandemia ha golpeado duramente al mercado de trabajo ${ }^{4}$, y esto afecta en gran medida al ODS 8 "Trabajo decente y crecimiento económico". En este sentido cabe señalar que mientras en el cuarto trimestre de 2019 la tasa de desempleo era del 13,8 \%, en el segundo trimestre de 2020 la tasa de desempleo aumentó hasta el 15,33\% $\%^{5}$, sin tener en cuenta los afectados por ERTE.

No a todos los sectores les ha afectado por igual, el impacto es mucho menor en aquellos sectores, aquellos trabajadores, que pueden realizar su trabajo a distancia, o los sectores que realizan trabajos esenciales que, aunque en condiciones muy difíciles, han mantenido e incluso incrementado su nivel de actividad.

En el caso de las personas con discapacidad la situación se agrava por su situación desigual de partida, la tasa de desempleo es superior en 10 puntos porcentuales a la que comparte la población sin discapacidad, lo que supone que, como otros segmentos vulnerables de la sociedad, muchos de sus integrantes pueden caer por debajo del umbral de la pobreza.

Si antes de la COVID-19 solo una de cada cuatro personas con discapacidad tenía empleo, según el estudio Efectos y consecuencias de la crisis de la Covid-19 entre las personas con discapacidad de ODISMET (Silván y Quífez, 2020), durante la pandemia un $12 \%$ de personas con discapacidad ha perdido su empleo y 3 de cada 5 temen perderlo próximamente.

No solo es problemática la destrucción de empleo, sino que también afecta a las posibilidades de encontrar un empleo futuro y llevar una vida autónoma, pudiendo cronificar la exclusión social de este colectivo.

También se ha visto afectada la educación en nuestro país, y por ende el ODS 4 "Educación de calidad". La decisión de cerrar los centros educativos, como refieren Ainara Zubillaga y Lucas Gortazar en su informe COVID-19 y educación: problemas, respuestas y escenarios, producirá un impacto negativo sobre el aprendizaje de todos los alumnos, pero será muy superior para los alumnos de entornos desfavorecidos.

Tener o no tener conexión a Internet, la mayor o menor calidad, disponer de dispositivos tecnológicos adecuados (Ethic, 2020), y en el caso de las personas con discapacidad, la necesidad de que estén concebidos con criterios de accesibilidad universal; y, además, contar con los apoyos adecuados, tanto técnicos como humanos, puede dar lugar o no a una brecha que acentúe la desigualdad existente de partida.

Cuando las situaciones de partida son desiguales, como las de los grupos vulnerables o en riesgo de exclusión social, más necesarias son políticas responsables alineadas con la Agenda 2030.

4. La OIT estima que la pérdida de horas de trabajo en el segundo semestre de 2020 (con respecto al cuarto trimestre de 2019) se eleva al 17,3 por ciento (495 millones de empleos equivalentes a tiempo completo) (OIT, 2020a).

5. Véase web INE (2020): https://www.ine.es/jaxiT3/Tabla.htm?t=4247 


\subsection{Necesidad de la sostenibilidad para la recuperación y la reconstrucción}

A pesar del desconocimiento generalizado en la sociedad sobre el ámbito de la sostenibilidad, existen ejemplos de buenas prácticas de aplicación de criterios sostenibles, alineados con la Agenda 2030, que debemos poner en valor.

Prueba de ello sería el ejemplo de BlackRock, la gestora mundial de inversiones más importante del mundo, que ha anunciado que retirará sus fondos de las empresas que no tengan en cuenta los ODS, en los próximos años ${ }^{6}$.

Otro ejemplo es Triodos Bank, uno de los bancos más sostenibles del mundo, donde solo se financian empresas que ponen la dignidad de las personas en el centro y buscan crear ese impacto positivo en los ámbitos social, cultural y medioambiental. O por citar un último ejemplo, el compromiso de IKEA por el cual todas sus actividades en el año 2030 serán circulares (propias de la economía circular7).

La crisis derivada de la pandemia COVID-19 ha puesto de manifiesto la necesidad de responder de forma unitaria desde el sector público, pero también desde la iniciativa privada, y fundamentalmente desde el sector empresarial.

Para afrontar la recuperación, la reconstrucción y la sostenibilidad, por tanto, la Agenda 2030 se presenta como una oportunidad en ese afán "de no dejar a nadie atrás". En este sentido, los ODS hacen indicaciones específicas en relación con los grupos en situación de vulnerabilidad social:

- Necesidad de plantear una educación de calidad, como indica el ODS 4 en su meta 4.5: "asegurar el acceso igualitario a todos los niveles de la enseñanza y la formación profesional para las personas vulnerables, incluidas las personas con discapacidad".

O en su meta 4.a: "construir y adecuar instalaciones educativas que tengan en cuenta las necesidades de los niños y las personas con discapacidad y las diferencias de género, y que ofrezcan entornos de aprendizaje seguros, no violentos, inclusivos y eficaces para todos".

- Empleo de calidad y sostenible. EI ODS 8 sobre trabajo decente y crecimiento económico plantea en la meta 8.2 el objetivo de "lograr niveles más elevados de productividad económica mediante la diversificación, la modernización tecnológica y la innovación”. Además, añade en la meta 8.4, "desvincular el crecimiento económico de la degradación del medio ambiente", y en su meta 8.5, "lograr el empleo pleno y productivo y el trabajo decente para todos, incluidos los jóvenes y las personas con discapacidad".

Pero además de la Agenda 2030, existen nuevas oportunidades sobre las que se debe asentar la recuperación y/o la reconstrucción sostenible. Estas se pueden encuadrar dentro de dos bloques fundamentales: la conservación del medio ambiente y la reducción de la desigualdad en los países y entre estos.

6. Carta anual (2020) de Larry Fink (presidente y consejero delegado BlackRock): https://www.blackrock.com/uk/individual/larry-fink-ceo-letter. 7. La economía circular es un concepto económico que se interrelaciona con la sostenibilidad, y cuyo objetivo es que el valor de los productos, los materiales y los recursos (agua, energía,...) se mantenga en la economía durante el mayor tiempo posible, reduciendo la generación de residuos y minimizando el consumo de recursos. 
Respecto a las oportunidades que brinda la conservación del medio ambiente, hay que hacer referencia: al desarrollo de la economía circular; la reducción del consumo y del desperdicio de materias primas; el fomento del consumo responsable de todos, no solo de las instituciones o de las empresas, también de los ciudadanos y de la sociedad en general; la utilización de energías renovables; la reducción de emisiones y la descarbonización para la sostenibilidad del planeta; etc. Pero, además, no podemos obviar que también es un valor añadido para las entidades y empresas, ya que la apuesta por este tema es un criterio de selección de proyectos para ser financiados, tanto en el ámbito público como privado.

En cuanto a la erradicación de la desigualdad, es fundamentalmente trabajar por la educación de calidad y la generación de empleos ligados a modelos de economía circular y a las energías renovables, impulsando la diversidad y la inclusión dentro de las organizaciones, sobre todo de los colectivos vulnerables. Asimismo, adquiere gran relevancia fomentar el emprendimiento, la I+D+l, la digitalización y la inversión en investigación y desarrollo.

Además de estas oportunidades, y específicamente respecto a las personas con discapacidad, sería fundamental que se vigilara el cumplimiento normativo de la cuota legal de reserva de puestos de trabajo para personas con discapacidad ya que facilitaría el acceso al empleo de un número importante de personas en riesgo de exclusión social. Asimismo, sería importante que las organizaciones y empresas empleen herramientas externas y certificaciones independientes que verifiquen la excelencia en la gestión ${ }^{8}$ sostenible.

La sostenibilidad debe ser vista como "una oportunidad y valor añadido". Ofrece una visión de necesidades y ambiciones globales que deben convertirse en soluciones, y para ello es necesario adoptar un modelo de crecimiento sobre la base de un propósito: lograr el progreso, pero de una forma sostenible, teniendo en cuenta los impactos en la sociedad y siempre, alineado con la Agenda 2030.

8. Certificación Bequal, Certificado EFR y Certificado SGE21. 


\section{Referencias bibliográficas}

AENOR (2010). Norma UNE-ISO 26000:2010(es). Guía de responsabilidad social.

Comisión Europea (2018). Comunicación de la comisión al parlamento europeo, al consejo, al comité económico y social europeo y al comité de las regiones. Estrategia renovada de la UE para 2011-2014 sobre la responsabilidad social de las empresas. Recuperado de https://bit.ly/33FpEBR.

Ethic (28 agosto, 2020). La economía española en tiempos de pandemia [Artículo en web]. Recuperado de https:// ethic.es/2020/08/la-economia-espanola-en-tiempos-de-pandemia/.

Fundación Gates (2020). COVID-19: una perspectiva mundial. Reporte Goalkeepers 2020. [s.l.]: Fundación Gates. Recuperado de https://www.gatesfoundation.org/goalkeepers/downloads/2020-report/report_a4_es.pdf.

Gobierno de España (2018). Plan de Acción para la implementación de la Agenda 2030: hacia una estrategia española de desarrollo sostenible. Madrid: Dirección General de Políticas de Desarrollo Sostenible. Recuperado de https://bit.ly/3qrpvvs.

Llano, J. (2018). El estado de la pobreza, $8^{\circ}$ informe AROPE. Seguimiento del indicador de riesgo de pobreza y exclusión social en España 2008-2017. Resumen ejecutivo. Madrid: Red Europea de Lucha contra la Pobreza y la Exclusión Social. Recuperado de https://bit.ly/39llxsG.

Llano, J. (2019). El estado de la pobreza, $9^{\circ}$ informe AROPE. Seguimiento del indicador de riesgo de pobreza y exclusión social en España 2008-2018. Resumen ejecutivo. Madrid: Red Europea de Lucha contra la Pobreza y la Exclusión Social. Recuperado de https://bit.ly/3mB7wk3.

Organización de Naciones Unidas (ONU) (25 septiembre, 2015). La Asamblea General adopta la Agenda 2030 para el desarrollo sostenible [Noticia en web]. Recuperado de https://www.un.org/sustainabledevelopment/ es/2015/09/la-asamblea-general-adopta-la-agenda-2030-para-el-desarrollo-sostenible/.

Organización Internacional del Trabajo (OIT) (2020a). Observatorio de la OIT: la COVID19 y el mundo del trabajo. Quinta edición. Estimaciones actualizadas y análisis. Recuperado de https://bit.ly/3IG8ome.

Organización Internacional del Trabajo (OIT) (2020b). Observatorio de la OIT: la COVID19 y el mundo del trabajo. Sexta edición. Estimaciones actualizadas y análisis. Recuperado de https://bit.ly/3gcaSrs.

Red Española del Pacto Mundial (7 abril, 2020). Los efectos de la COVID-19 en los ODS [Artículo en web]. Recuperado de https://bit.ly/219yEaM.

Silván, C. y Quífez, L. (2020). Efectos y consecuencias de la crisis de la COVID-19 entre las personas con discapacidad. Madrid: Fundación Once. Recuperado de https://bit.ly/37Ae6Rn.

Zubillaga, A. y Gortazar, L. (2020). COVID-19 y educación I: problemas, respuestas y escenarios. Documento técnico de análisis de la situación educativa derivada de la emergencia sanitaria. Madrid. Fundación COTEC. Recuperado de https://online.flippingbook.com/view/967738/. 